\title{
LATE PLEISTOCENE AND HOLOCENE BISON OF THE COLORADO PLATEAU
}

\author{
Jeff M. Martin,* Rachel A. Martin, and Jim I. Mead \\ Wildlife and Fisheries Sciences, Texas AENM University, College Station, TX 77843 (JMM) \\ Ecosystem Science and Management, Texas AEM University, College Station, TX 77843 (RAM) \\ The Mammoth Site, Hot Springs, SD 57747 (JIM) \\ Don Sundquist Center of Excellence in Paleontology, East Tennessee State University Gray Fossil Site and Museum, \\ Gray, TN 37615 (JMM, RAM, JIM) \\ *Correspondent: jeff.m.martin@exchange.tamu.edu
}

\begin{abstract}
Fossils of Bison (bison) are scarce on the Colorado Plateau, especially in and around the Greater Grand Canyon Region. Because of poor preservation and collection biases in the region, various resource managers have erroneously designated bison a nonnative and human-(re)introduced species. This decision directly impacts an extant herd of approximately 400 bison that periodically meander onto Grand Canyon National Park lands from neighboring U.S. Forest Service and State of Arizona lands. We re-examined and verified paleozoological museum specimens of this large mammal confirming a prehistoric and early historic presence of Bison on the Colorado Plateau. Our findings indicate that Bison bison should be considered a native species on the Colorado Plateau because they have a nearly continuous record of inhabitance in the region. This record is supported by 74 Bison-bearing fossil, subfossil, and historical localities since the latest Pleistocene.

Resumen-Los fósiles de Bison (bisontes) son escasos en la meseta del Colorado, especialmente dentro y alrededor de la región del Gran Cañón. Debido a la pobre preservación de los fósiles y parcialidad en las colecciones de la región, varios administradores de recursos naturales han denominado equivocadamente bisontes como especies exóticas e introducidas por humanos. Esta decisión impacta directamente a la población actual de cerca de 400 bisontes que a veces traspasan a los terrenos del Servicio Forestal de E.E.U.U. y del Estado de Arizona, hasta los terrenos del Parque Nacional del Gran Cañón. Re-examinamos y verificamos especímenes paleozoológicos de museos de este gran mamífero, confirmando la presencia prehistórica e histórica temprana de Bison en la meseta del Colorado. Nuestros resultados indican que Bison bison debe ser considerado como especie nativa en la meseta del Colorado por su casi continuo registro de habitación dentro de la región. Este record es respaldado por 74 fósiles y subfósiles relacionados con Bison, y por localidades históricas desde el Pleistoceno tardío.
\end{abstract}

Perhaps no other extant animal in North America possesses the cultural, spiritual, ecological, economic, political, and natural history attributes that are emblematic of the North American bison (Bison bison; Artiodactyla, Bovidae, Bovini; Isenberg, 1997; Sanderson et al., 2008). Certainly, no other animal boasts the story of surviving the brink of extinction twice. This story does not stop at the North American plains subspecies (Bison bison bison), but it also applies to the North American woods bison subspecies (Bison bison athabascae) along with the European bison species (Bison bonasus; wisent). First, Bison survived the megafaunal extinction at the end of the Pleistocene approximately 11,700 calendar years Before Present (cal yr BP) and, in North America, the genus outlived mammoths (Mammuthus), mastodons (Mammut), horses (Equus), ground sloths (Megalonyx et al.), and other megafauna while coexisting with early Americans
(Paleoindians; Koch and Barnosky, 2006). Second, Bison survived the threat of Americans of European descent, who purposefully hunted the bison nearly to extinction, during the late 1800s Common Era (Hornaday, 1889).

The most recent comprehensive analysis of Bison distribution in continental North America illustrates an apparent geographic 'gap' of bison records for much of the arid Southwest, especially on the Colorado Plateau (CP; Mc Donald, 1981; Fig. 1). In the western United States, there are well-documented occurrences of Quaternary-aged Bison in physiographic provinces neighboring the CP, such as the Great Basin (Jennings, 1978; Scott and Cox, 2008), southern and middle Rocky Mountains (McDonald, 1981), Wyoming Basin (McDonald, 1981), Arizona deserts (Agenbroad and Haynes, 1975; Wolff, 2013), Bonneville Basin (Madsen et al., 2001), and Rio Grande Rift basin (Harris, 2014). 


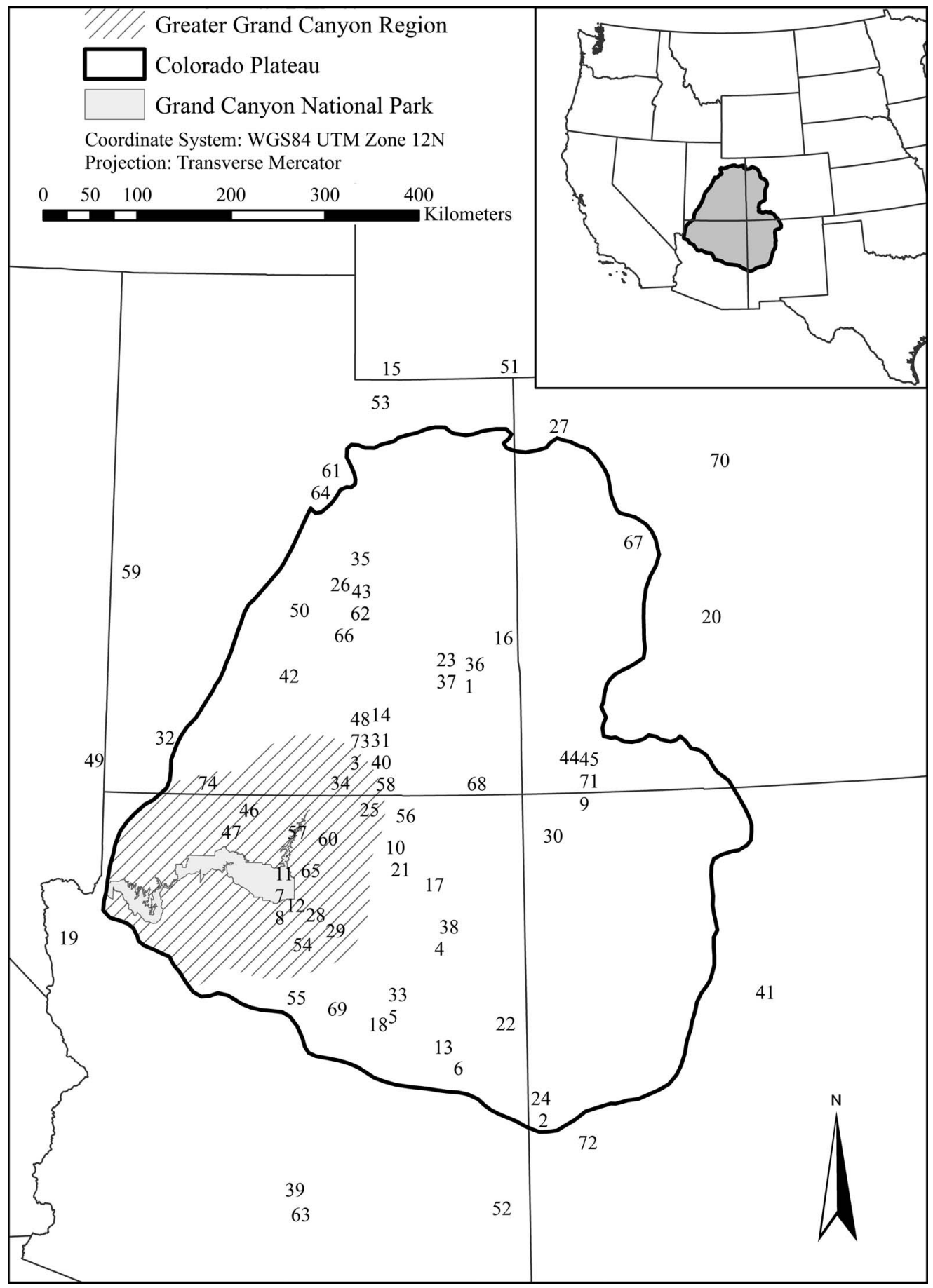

FIG. 1-Map of Bison localities (placement is approximate) on the Colorado Plateau (CP, heavy line) and surrounding region. Locality numbers refer to Table 1. Hashed line represents our definition of the Greater Grand Canyon Region (GGC, see MATERIALS AND Methods section) surrounding Grand Canyon National Park (GCNP, shaded area).

In recent years, bison from Arizona's House Rock Valley in Arizona have dispersed onto the Kaibab Plateau on the North Rim of the Grand Canyon National Park (NP). Resource managers subsequently questioned the nativity of Bison on the CP and concluded, from lack of evidence and limited tangential environmental assessments (Reimondo, 2012; Huffer, 2013), that bison are not native to the area. These prior studies made no examination of specimens in collections but relied upon published literature. Thus, it is necessary to complete a comprehensive assessment of unpublished accounts of Bison remains from paleontological and archaeological sites that are preserved in local museum collections.

Here, we synthesize the paleontological and archaeological localities known to contain Bison from the Grand Canyon and the surrounding $\mathrm{CP}$ along with a few just off 
the plateau for adjacent references. Our data set includes published and unpublished accounts and unstudied museum specimens along with those records archived in database systems that have recently emerged. Our goal is to address two main questions: 1) when and 2) where did Bison occur on the CP, especially within the Grand Canyon NP and the Greater Grand Canyon Region (as defined in Materials and Methods)? We address these questions by assessing, and in some cases describing, museum specimens of Bison from 74 localities on and around the CP (Table 1; Fig. 1). Details about each locality are in the thesis by Martin (2014); we provide only pertinent data herein.

BACKground-Today, there are two, albeit disputed, subspecies of Bison (Cronin et al., 2013): 1) Bison bison bison are historically found in the Great Plains and elsewhere throughout much of North America, and 2) Bison bison athabascae are historically found north of $49^{\circ} \mathrm{N}$ latitude in Canada and Alaska. This project does not address subspecies or species but focuses on the animal at the generic level. In all, Bison bison, Bison antiquus, and Bison latifrons have been reported on the $\mathrm{CP}$ and in the surrounding provinces (Table 1).

The historical chronicle of bison in the Southwest is complex and confusing. Northern Arizona has scarce historical records, in general, but the Spanish reported a small herd of Bison in the 16th century in east-central New Mexico and adjacent to the CP (Reed, 1952). Native Americans in the $1200 \mathrm{~s} \mathrm{AD}$ and earlier created pictographs and petroglyphs on the walls of Kanab Creek near Kanab, Utah (among other places; Malotki and Wallace, 2011). However, the ideas, memories, and thoughts that inspired these renderings could conceivably have travelled great distances and well beyond the actual zoogeographic distributions, which brings into question the idea that the pictographs and petroglyphs represent local occurrences of bison. A few unrelated, documented occurrences confirm that bison frequented the CP near the Greater Grand Canyon Region in small but selfsustaining herds that most likely had relatively large home ranges to endure the low carrying capacity of the region (Seager et al., 2007).

In 1905, Charles J. "Buffalo" Jones brought bison to the Kaibab Plateau on the North Rim of the Grand Canyon NP (Hoffmeister, 1986) and crossbred them with Bos taurus to create a hardy livestock animal he called "cattelo." In 1906, when Congress established the Grand Canyon Game Preserve, they listed bison as one of the wildlife species that should be maintained on the Kaibab Plateau (unpublished report to U.S. Congress, Protection of Wild Animals in the Grand Canyon Forest Preserve). The Kaibab Plateau was described as "ideal for buffalo [Bison], deer and other wild game" and was "to be recognized as a breeding place therefore" (Wakeling, 2006:25). Most importantly, "the Preserve was created on
28 November 1906 by President Theodore Roosevelt and is still in effect. It predates the establishment of the [Kaibab] National Forest [in 1909], Grand Canyon National Park [in 1919], and the Arizona Game and Fish Commission [in 1913]" (Wakeling, 2006:25). In 1909, these bison were relocated east to the House Rock Valley (Marble Platform) because of the creation of the Kaibab National Forest when C.J. Jones moved all but 15-20 animals out of the area. The 15-20 remaining bison became property of James T. "Uncle Jim" Owens and, by 1927, the herd had increased to 98 individuals and was purchased by the State of Arizona via the Arizona Game and Fish Department. Over the past half-century, introduced 'purebred' Bison bison from Oklahoma and Montana have been added to the bison herd to improve its natural resilience (see discussions in Hoffmeister, 1986; Wakeling, 2006).

Throughout the Holocene, Bison abundances spatially varied over most of North America possibly because of hunting and intraspecific competition; yet, the population steadily increased in the Great Plains until European contact when their abundance probably reached their apex (Flores, 1991). This increase in bison might have occurred because of a few conditions including limited trophic-equivalent competition (Flores, 1991); reduced abundance and diversity of presumed predators since the Pleistocene extinction of the dire wolf (Canis dirus), American lion (Panthera atrox), and the giant short-faced bear (Arctodus simus; Flores, 1991); and cooler, wetter climate conditions, which are favorable for grass growth (Wisely et al., 2008; Craine, 2013; Craine et al., 2013). Thus, the observations made by early western explorers (Hornaday, 1889) are likely misleading or at least poorly representative of Bison abundance.

Furthermore, earlier in the Holocene, Bison were less abundant in the Great Plains and were intermittently present in the Southwest (Broughton et al., 2008). There are potentially multiple factors contributing to the seemingly poor fossil record of Bison during the Late Pleistocene and Holocene on the CP. Yet, the region is incompletely studied by Quaternary paleontologists and zooarchaeologists compared with the neighboring provinces, most likely because of its remoteness.

Materials and Methods-The Colorado Plateau is an immense physiographic province between the Colorado Rocky Mountains and the Great Basin Desert (Blakey and Ranney, 2008). The Grand Canyon is defined as the geological gorge that incises the Colorado Plateau (Ranney, 2012) and is immediately surrounded by Grand Canyon NP. We define the Greater Grand Canyon Region as the ecosystem of Grand Canyon NP adjacent to the Colorado River corridor and the plateaus immediately rimming the Grand Canyon gorge. This includes the Coconino, Hualapai, Kaibab, Kanab, Uinkaret, and Shivwits plateaus and the Marble Platform. Thus, we also extend the Greater Grand Canyon Region to approximately $80 \mathrm{~km}$ beyond the borders of Grand Canyon NP to encompass the habitats of each plateau (Fig. 1). 
TABLE 1-Summary table of Bison localities from the Colorado Plateau region. Key refers to number and location in Figure 1. Mean age in calendar years Before Present (cal yr BP) calibrated by IntCal13 (Reimer et al., 2013). References other than primary literature include GRCA collection (Grand Canyon National Park collections), MNA (Museum of Northern Arizona), AZSite (Arizona's Cultural Resource Inventory), NeotomaDB (Neotoma Paleoecology Database), and NeoMap (Neogene Mammal Mapping Portal). “-” indicates no data. Empty cells indicate no information is applicable.

\begin{tabular}{|c|c|c|c|c|c|c|}
\hline Key & Locality name & $\begin{array}{l}\text { Database } \\
\text { reference }\end{array}$ & $\begin{array}{l}\text { Mean age } \\
(\text { cal yr BP) }\end{array}$ & Cited age & Dating method & References \\
\hline 1 & The Neck ${ }^{\mathrm{a}}$ & 42SA8502 & 435 & $\begin{array}{l}\text { Common Era } \\
\text { (AD) } 1235- \\
\text { 1415; AD 1425- } \\
\text { 1655; AD 1425- } \\
1655 \text {; AD } 1485- \\
1795\end{array}$ & $\begin{array}{l}\text { Pollen and } \\
\text { charcoal }\end{array}$ & $\begin{array}{l}\text { Osborn et al., } \\
1995\end{array}$ \\
\hline 2 & Ada Mesa ${ }^{a}$ & $\begin{array}{l}\text { NA20657 Lots } 50 \\
\quad \& 52\end{array}$ & 1,100 & $\begin{array}{l}\text { Georgetown/ San } \\
\text { Francisco AD } \\
600-700+; \\
\text { Tularosa AD } \\
1100+ \\
\text { (Mogollon } \\
\text { Culture) }\end{array}$ & $\begin{array}{l}\text { Radiocarbon, } \\
\text { lithics, and } \\
\text { ceramics }\end{array}$ & - \\
\hline 3 & Alcove Spring & 42SA8512 & 453 & $\mathrm{AD} 1345-1650$ & $\begin{array}{r}\text { Pollen and } \\
\text { charcoal }\end{array}$ & $\begin{array}{l}\text { Osborn et al., } \\
1995\end{array}$ \\
\hline 4 & Awatovi $^{\mathrm{a}}$ & $\begin{array}{l}\text { NeotomaDB } \\
\text { 5910; NeoMap } \\
2332\end{array}$ & 850 & $\begin{array}{l}250-1,450 \mathrm{cal} \mathrm{yr} \\
\text { BP }\end{array}$ & & $\begin{array}{l}\text { Montgomery et } \\
\text { al., 1949; } \\
\text { Lawrence, } \\
\text { 1951; } \\
\text { Agenbroad and } \\
\text { Haynes, } 1975\end{array}$ \\
\hline 5 & AZ J:14:356 $6^{\mathrm{a}}$ & & 225 & 0 to $450 \mathrm{cal} \mathrm{yr} \mathrm{BP}$ & Firepit charcoal & AZSite \\
\hline 6 & AZ $P: 8: 3^{\mathrm{a}}$ & & 1,325 & $\begin{array}{l}1,200-1,450 \mathrm{cal} \mathrm{yr} \\
\text { BP }\end{array}$ & $\begin{array}{l}\text { Lithics and } \\
\text { ceramics }\end{array}$ & AZSite \\
\hline 7 & $\begin{array}{l}\text { Mather } \\
\text { Campground }\end{array}$ & B:16:0461 & 50 & $50 \pm 30 \mathrm{yr}$ & Bison phalanx & This study \\
\hline 8 & B:16:105 & GRCA 69396 & 1,050 & $\begin{array}{l}927 \text { cal yr BP; } \\
1000 \text { yr BP } \\
\text { (lithics) }\end{array}$ & $\begin{array}{l}\text { Bison lipids on } \\
\text { ceramics }\end{array}$ & $\begin{array}{l}\text { Downum et al., } \\
2011\end{array}$ \\
\hline 9 & Badger House & $\begin{array}{l}\text { NeotomaDB } \\
\text { 1453; NeoMap } \\
1268\end{array}$ & 814 & $650-1,100$ yr BP & ${ }^{14} \mathrm{C}$ on unknown & $\begin{array}{l}\text { Hayes and } \\
\quad \text { Lancaster, } 1975\end{array}$ \\
\hline 10 & Badger Spring & $\begin{array}{l}\text { MNA.Loc.112-0; } \\
\text { MNA Ariz } \\
\text { D:5:13; } \\
\text { NA10924 }\end{array}$ & 10,000 & $7,500-9,500$ yr BP & Lithic technology & $\begin{array}{l}\text { Hesse et al., 1999; } \\
\text { MNA collection }\end{array}$ \\
\hline 11 & Battleship Rock & & 12,000 & & $\begin{array}{l}\text { Faunal } \\
\text { assemblage } \\
\text { (Equus sp.) }\end{array}$ & GRCA collection \\
\hline 12 & Beamer's Cabin & AZ C:13:0004 GC & 555 & $410-700$ cal yr BP & $\begin{array}{l}\text { Association of } \\
\text { ceramics }\end{array}$ & $\begin{array}{l}\text { Jones, 1986; } \\
\text { Huffer, } 2013\end{array}$ \\
\hline 13 & Bear Ruin & AZ P:16:1 & 625 & $550-700$ cal yr BP & Ceramics & $\begin{array}{l}\text { Haury, 1940; } \\
\text { Agenbroad and } \\
\text { Haynes, 1975; } \\
\text { Lightfoot and } \\
\text { Feinman, } 1982\end{array}$ \\
\hline 14 & Bechan Cave & $\begin{array}{l}\text { NAU QSP Site } \\
\text { 872; GLCA } \\
\text { Accession } 81\end{array}$ & 15,182 & $\begin{array}{l}11,670 \pm 300 \\
13,505 \pm 580 \\
\text { yr BP }\end{array}$ & Mammuthus dung & $\begin{array}{l}\text { Mead and } \\
\text { Agenbroad, } \\
\text { 1992; Kropf et } \\
\text { al., } 2007\end{array}$ \\
\hline 15 & $\begin{array}{l}\text { Bessie Bottom } \\
\text { Site }\end{array}$ & $\begin{array}{l}\text { 48UT1186; } \\
\text { NeotomaDB } \\
4810\end{array}$ & 985 & $\begin{array}{l}910 \pm 80 ; 1,170 \\
\quad \pm 60 \mathrm{yr} \mathrm{BP}\end{array}$ & - & McKern, 1988 \\
\hline
\end{tabular}




\begin{tabular}{|c|c|c|c|c|c|c|}
\hline Key & Locality name & $\begin{array}{l}\text { Database } \\
\text { reference }\end{array}$ & $\begin{array}{l}\text { Mean age } \\
(\text { cal yr BP) }\end{array}$ & Cited age & Dating method & References \\
\hline 16 & Bison Alcove & $\begin{array}{l}\text { 42GR538 (ARCH } \\
\text { 115); } \\
\text { MNA.Loc. } \\
\text { 9144; } \\
\text { NeotomaDB } \\
\text { 6290; NeoMap } \\
\text { 2958 }\end{array}$ & 445 & $\begin{array}{l}355 \pm 60 ; 405 \pm \\
65 \text { yr BP }\end{array}$ & $\begin{array}{l}\text { Bison horn and } \\
\text { hoof }\end{array}$ & Mead et al., 1991 \\
\hline 17 & Black Mesa ${ }^{a}$ & $\begin{array}{l}\text { D:11:2062; } \\
\text { D:7:0713; } \\
\text { NeotomaDB } \\
6010 \& 5980\end{array}$ & 1,302 & $\begin{array}{l}885 \pm 72 ; 1,673 \\
\quad \pm 117 \mathrm{cal} \mathrm{yr} \mathrm{BP}\end{array}$ & - & Leonard, 1989 \\
\hline 18 & $\begin{array}{l}\text { Canyon Diablo } \\
\text { Dam }\end{array}$ & $\begin{array}{l}\text { NA8793.Lot.1 } \\
\quad \# 7136\end{array}$ & 975 & Pueblo II & - & - \\
\hline 19 & Catclaw Cave & AZ F:2:1(ASM) & 7,225 & $\begin{array}{c}12,000 \mathrm{yr} \mathrm{BP}- \\
1500 \mathrm{AD}\end{array}$ & $\begin{array}{l}\text { Bison bison with } \\
\text { assorted fishes } \\
\text { associated with } \\
\text { lithics and } \\
\text { ceramics }\end{array}$ & AZSite \\
\hline 20 & $\begin{array}{l}\text { Cement Creek } \\
\text { Cave }\end{array}$ & - & 22,585 & $\begin{array}{l}43,330 \pm \\
\quad 760 ; 1,120 \pm 40 \\
\text { yr BP }\end{array}$ & $\begin{array}{l}\text { Marmota } \\
\text { flaviventris }\end{array}$ & Emslie, 2002 \\
\hline 21 & $\begin{array}{l}\text { Charley Day } \\
\text { Spring }\end{array}$ & $\begin{array}{l}\text { NA1898; } \\
\text { MNA.Loc. 149- } \\
1\end{array}$ & 14,000 & Rancholabrean & $\begin{array}{l}\text { Faunal } \\
\text { assemblage }\end{array}$ & $\begin{array}{l}\text { MNA collection; } \\
\text { Lindsay and } \\
\text { Tessman, 1974; } \\
\text { Agenbroad and } \\
\text { Haynes, } 1975\end{array}$ \\
\hline 22 & $\begin{array}{l}\text { Cottonwood Seep } \\
\text { Spring }^{\mathrm{a}}\end{array}$ & $\begin{array}{l}\text { NA14674.Lot.955 } \\
\text { and Lot.985 \& } \\
\text { GSPS6 }\end{array}$ & 975 & Pueblo II & Lithics & MNA collection \\
\hline 23 & Cowboy Cave & $\begin{array}{l}\text { 45WN420; } \\
\text { NeotomaDB } \\
\text { 9761; NeoMap } \\
2048\end{array}$ & 14,519 & $\begin{array}{l}11,020 \pm 180 \\
\quad 13,040 \pm 440 \\
\text { yr BP }\end{array}$ & Bison dung & $\begin{array}{l}\text { Hansen, 1980; } \\
\text { Lucias, 1980; } \\
\text { Mead and } \\
\text { Agenbroad, } \\
1992\end{array}$ \\
\hline 24 & Coyote Creek & NA14064 & 750 & $\begin{array}{l}\text { Tularosa phase of } \\
\text { Anasazi } \\
\text { (Pueblo III) }\end{array}$ & $\begin{array}{l}\text { Lithics and } \\
\text { buildings }\end{array}$ & MNA collection \\
\hline 25 & Dust Devil Cave & $\begin{array}{l}\text { NA7613.Lot. } \\
\text { 1066.A9V6; } \\
\text { MNA.UT:V: } \\
\text { 13:160 }\end{array}$ & 975 & $\begin{array}{l}\text { Kayenta culture } \\
\text { (Pueblo II) }\end{array}$ & Ceramics & - \\
\hline 26 & $\begin{array}{l}\text { Ephraim (Witch's } \\
\text { Knoll) }\end{array}$ & $\begin{array}{l}\text { NeotomaDB: } \\
\quad 7683\end{array}$ & 850 & $\begin{array}{c}664 \pm 6 ; 1020 \pm \\
22 \text { cal yr BP }\end{array}$ & - & $\begin{array}{l}\text { Gillin and Allen, } \\
1941\end{array}$ \\
\hline 27 & $\begin{array}{l}\text { Fort Davy } \\
\text { Crockett }\end{array}$ & $\begin{array}{l}\text { 5MF605; } \\
\text { NeotomaDB } \\
\text { 5210; NeoMap } \\
1165\end{array}$ & 490 & $\begin{array}{c}50 \pm 1 ; 927 \pm 3 \\
\text { cal yr BP }\end{array}$ & - & $\begin{array}{c}\text { Sharrock, 1966; } \\
\text { Walker, } 1983\end{array}$ \\
\hline 28 & Furnace Flats & AZ C:13:0010 GC & 995 & $\mathrm{AD} 641-1270$ & $\begin{array}{l}\text { Association of } \\
\text { ceramics }\end{array}$ & $\begin{array}{l}\text { Jones, 1986; } \\
\text { Huffer, } 2013\end{array}$ \\
\hline 29 & Grand Falls & $\begin{array}{c}\text { MNA V8301 } \\
\text { Loc.1104 }\end{array}$ & 50 & $\begin{array}{l}50 \pm 30 \\
\quad \text { radiocarbon } \\
\quad \text { years }\end{array}$ & Bison rib head & This study \\
\hline 30 & Gray Water Wash & MNA.Loc.358-0 & 14,000 & Rancholabrean & - & - \\
\hline
\end{tabular}


TABLE 1- Continued.

\begin{tabular}{|c|c|c|c|c|c|c|}
\hline Key & Locality name & $\begin{array}{l}\text { Database } \\
\text { reference }\end{array}$ & $\begin{array}{l}\text { Mean age } \\
(\text { cal yr BP) }\end{array}$ & Cited age & Dating method & References \\
\hline 31 & Grobot Grotto & $\begin{array}{l}\text { NAU QSP Site } \\
\text { 878; GLCA } \\
\text { Accession } 82\end{array}$ & 27,384 & $\begin{array}{l}18,528 \pm 137 \\
33,540 \pm 2836 \\
\text { cal yr BP }\end{array}$ & Bison dung & $\begin{array}{l}\text { Mead and } \\
\text { Agenbroad, } \\
\text { 1989, 1992; } \\
\text { Withers and } \\
\text { Mead, 1993 }\end{array}$ \\
\hline 32 & Hamblin Springs & NeoMap 6329 & 5,850 & Holocene & - & - \\
\hline 33 & Homolovi I; & $\begin{array}{l}\text { AZ J:14:3; MNA } \\
\text { AZ J:14:8; } \\
\text { NA952 }\end{array}$ & 575 & $\begin{array}{l}\text { Pueblo IV (AD } \\
1300-1450)\end{array}$ & $\begin{array}{l}\text { Lithic technology } \\
\text { and ceramic } \\
\text { styles }\end{array}$ & AZSite \\
\hline 34 & Hooper's Hollow & $\begin{array}{l}\text { NAU QSP Site } \\
\text { 873; GLCA } \\
\text { Accession } 82\end{array}$ & 22,783 & $\begin{array}{l}22,783 \pm 405 \mathrm{cal} \\
\quad \text { yr BP }\end{array}$ & Bison dung & $\begin{array}{l}\text { Mead and } \\
\text { Agenbroad, } \\
\text { 1989, 1992; } \\
\text { Withers and } \\
\text { Mead, } 1993\end{array}$ \\
\hline 35 & $\begin{array}{l}\text { Huntington } \\
\text { Reservoir } \\
\text { Sinkhole }\end{array}$ & $\begin{array}{l}\text { NeotomaDB } \\
5737 \text {; NeoMap } \\
1953\end{array}$ & 12,668 & $\begin{array}{l}12,668 \pm 296 \mathrm{yr} \\
\mathrm{BP}\end{array}$ & $\begin{array}{l}\text { Mammut bone } \\
\text { amino acid }\end{array}$ & $\begin{array}{l}\text { Miller, 1987; } \\
\text { Gillette and } \\
\text { Madsen, } 1992\end{array}$ \\
\hline 36 & Island in the Sky & 一 & 975 & Pueblo II & Carving styles & 一 \\
\hline 37 & Jim Walter's Cave & - & 14,519 & $\begin{array}{l}11,020 \pm 180 \\
13,040 \pm 440 \\
\text { yr BP }\end{array}$ & $\begin{array}{l}\text { Associated Bison } \\
\text { dung in } \\
\text { Cowboy Cave }\end{array}$ & Lucias, 1980 \\
\hline 38 & Keams Canyon & - & 14,000 & Rancholabrean & - & $\begin{array}{l}\text { Hay, 1927; } \\
\text { Lindsay and } \\
\text { Tessman, 1974; } \\
\text { Agenbroad and } \\
\text { Haynes, } 1975\end{array}$ \\
\hline 39 & Las Colinas & - & 575 & $\begin{array}{l}\text { Hohokam } \\
\text { (Pueblo IV) }\end{array}$ & - & $\begin{array}{l}\text { Johnson, 1974; } \\
\text { Agenbroad and } \\
\text { Haynes, 1975; } \\
\text { Teague and } \\
\text { Deaver, } 1989\end{array}$ \\
\hline 40 & Mammoth Alcove & $\begin{array}{l}\text { NAU QSP Site } \\
\text { 875; GLCA } \\
\text { Accession } 82\end{array}$ & 20,082 & $\begin{array}{l}16,630 \pm 280 \mathrm{yr} \\
\mathrm{BP}\end{array}$ & Mammuthus dung & $\begin{array}{l}\text { Mead and } \\
\text { Agenbroad, } \\
1992\end{array}$ \\
\hline 41 & $\begin{array}{l}\text { Martinez Gravel } \\
\text { Pit }\end{array}$ & NeotomaDB 6073 & 61,655 & $\begin{array}{l}13,341 \pm 30 \\
110,000 \mathrm{cal} \mathrm{yr} \\
\text { BP }\end{array}$ & $\begin{array}{l}\text { Bounding } \\
\text { formations }\end{array}$ & $\begin{array}{l}\text { Lucas et al., 1988; } \\
\text { Smartt et al., } \\
1991\end{array}$ \\
\hline 42 & Marysvale & NeotomaDB 7682 & 978 & $\begin{array}{c}603 \pm 37 ; 1379 \pm \\
12 \text { cal yr BP }\end{array}$ & $\begin{array}{l}\text { Unspecified } \\
\text { remains }\end{array}$ & $\begin{array}{l}\text { Gillin and Allen, } \\
1941\end{array}$ \\
\hline 43 & $\begin{array}{l}\text { Mastodon } \\
\text { Sinkhole }\end{array}$ & $\begin{array}{l}\text { NeoMap 6315; } \\
\text { 42EM231V }\end{array}$ & 14,000 & Rancholabrean & $\begin{array}{l}\text { Faunal } \\
\text { assemblage }\end{array}$ & 一 \\
\hline 44 & $\begin{array}{l}\text { Mesa Verde site } \\
\quad 866\end{array}$ & $\begin{array}{l}\text { NeoMap 1286; } \\
\text { NeotomaDB } \\
5320\end{array}$ & 750 & $\begin{array}{c}778 \pm 18 ; 856 \pm \\
37 \text { cal yr BP }\end{array}$ & - & Anderson, 1966 \\
\hline 45 & $\begin{array}{l}\text { Mesa Verde site } \\
\quad 875\end{array}$ & $\begin{array}{l}\text { NeoMap 1284; } \\
\text { NeotomaDB } \\
5318\end{array}$ & 975 & $\begin{array}{c}856 \pm 37 ; 927 \pm \\
3 \text { cal yr BP }\end{array}$ & - & Anderson, 1966 \\
\hline 46 & Kanab Creek & $\begin{array}{l}\text { NA8960. } \\
\quad \text { NA9074.Lot.2 }\end{array}$ & 1,075 & $\begin{array}{l}850-1300 \mathrm{cal} \mathrm{yr} \\
\text { BP }\end{array}$ & - & GRCA collections \\
\hline 47 & Mt. Trumbull ${ }^{\mathrm{a}}$ & $\begin{array}{l}\text { NA9074 S-213; } \\
\text { MNA.AZ.B:1:23 }\end{array}$ & 975 & $\begin{array}{l}850-1100 \mathrm{cal} \mathrm{yr} \\
\text { BP }\end{array}$ & - & MNA collections \\
\hline 48 & Oak Haven & $\begin{array}{l}\text { GLCA Accession } \\
\text { 82; NAU QSP } \\
\text { Site } 881\end{array}$ & 11,958 & $\begin{array}{l}9,180 \pm 100 \\
\quad 11,690 \pm 120 \\
\quad \text { yr BP }\end{array}$ & Quercus gambelii & $\begin{array}{l}\text { Mead and } \\
\text { Agenbroad, } \\
\text { 1989, 1992; } \\
\text { Withers and } \\
\text { Mead, 1993 }\end{array}$ \\
\hline
\end{tabular}


TABLE 1- Continued.

\begin{tabular}{|c|c|c|c|c|c|c|}
\hline Key & Locality name & $\begin{array}{l}\text { Database } \\
\text { reference }\end{array}$ & $\begin{array}{l}\text { Mean age } \\
(\text { cal yr BP) }\end{array}$ & Cited age & Dating method & References \\
\hline 49 & O'Malley Shelter & $\begin{array}{l}\text { NeoMap 2774; } \\
\text { NeotomaDB } \\
6162\end{array}$ & 6,739 & $\begin{array}{l}7,100 \pm 190 \mathrm{yr} \\
\mathrm{BP}\end{array}$ & Charcoal & $\begin{array}{l}\text { Fowler et al., } \\
1973\end{array}$ \\
\hline 50 & Pharo Village & $\begin{array}{l}\text { NeoMap 2848; } \\
\text { Netoma } 6191\end{array}$ & 681 & $760 \pm 80$ yr BP & Wood & Marwitt, 1968 \\
\hline 51 & Pine Springs & $\begin{array}{l}\text { NeotomaDB } \\
\quad 4820 ; 48 S W 101\end{array}$ & 8,560 & $\begin{array}{l}7,695 \pm 195 \mathrm{yr} \\
\mathrm{BP}\end{array}$ & Bone collagen & $\begin{array}{l}\text { Sharrock, 1966; } \\
\text { Frison, } 1978\end{array}$ \\
\hline 52 & Point of Pines & $\begin{array}{l}\text { NeoMap } 2236 \text { \& } \\
\text { 2242; } \\
\text { NeotomaDB } \\
5866\end{array}$ & 573 & $\begin{array}{c}543 \pm 28 ; 595 \pm \\
35 \text { cal yr BP }\end{array}$ & - & $\begin{array}{l}\text { Stein, 1963; } \\
\text { Agenbroad and } \\
\text { Haynes, } 1975\end{array}$ \\
\hline 53 & Porcupine Cave & NeotomaDB 7680 & 542 & $510 \pm 75$ yr BP & $\begin{array}{l}\text { Ursus americanus } \\
\text { bone }\end{array}$ & Heaton, 1988 \\
\hline 54 & Red Horse Wash ${ }^{\mathrm{b}}$ & $\begin{array}{l}\text { NA9528.Lot.4 \& } \\
\text { Lot.1 (NA } \\
5164)\end{array}$ & 975 & $\begin{array}{l}\text { Cohonina/ } \\
\text { Anasazi/ } \\
\text { Archaic } \\
\text { (Pueblo II) }\end{array}$ & Ceramics & MNA collections \\
\hline 55 & Ridge Ruin & & 750 & $\begin{array}{l}\text { Hohokam } \\
\quad \text { (Pueblo III) }\end{array}$ & Lithics & $\begin{array}{c}\text { Agenbroad and } \\
\text { Haynes, } 1975\end{array}$ \\
\hline 56 & Sand Dune Cave & $\begin{array}{l}\text { NA7523.Lot. } \\
\text { 133:2183 } \\
\text { (MNA.UT.V:13 } \\
\text { 74) }\end{array}$ & 5,650 & $\begin{array}{l}\text { Navajo or } \\
\text { Basketmaker I/ } \\
\text { III }\end{array}$ & - & MNA collections \\
\hline 57 & Sandblast Cave & $\begin{array}{l}\text { NeoMap 4875; } \\
\text { NeotomaDB } \\
7710\end{array}$ & 13,704 & $\begin{array}{l}13,110 \pm 680 \mathrm{yr} \\
\mathrm{BP}\end{array}$ & $\begin{array}{l}\text { Associated } \\
\quad \text { Oreamnos dung }\end{array}$ & $\begin{array}{l}\text { Emslie, 1987, } \\
\text { 1988; Mead } \\
\text { and } \\
\text { Agenbroad, } \\
\text { 1989, } 1992\end{array}$ \\
\hline 58 & Shrub Ox Alcove & $\begin{array}{l}\text { GLCA Accession } \\
\text { 82; NAU QSP } \\
\text { Site } 882\end{array}$ & 15,003 & $\begin{array}{l}12,690 \pm 180 \mathrm{yr} \\
\mathrm{BP}\end{array}$ & $\begin{array}{l}\text { Quercus twig with } \\
\text { Bison dung } \\
\text { associated }\end{array}$ & $\begin{array}{l}\text { Mead and } \\
\text { Agenbroad, } \\
\text { 1989, 1992; } \\
\text { Withers and } \\
\text { Mead, } 1993\end{array}$ \\
\hline 59 & Smith Creek Cave & $\begin{array}{l}\text { NeotomaDB } \\
\text { 4684; 26WP46 }\end{array}$ & 27,267 & $\begin{array}{l}\text { Reddish-Brown } \\
\text { Silt Zone } \\
(12,600-35,000 \\
\text { yr BP })\end{array}$ & ${ }^{14} \mathrm{C}$ on unknown & $\begin{array}{l}\text { Bryan, 1979; } \\
\text { Mead et al., } \\
\text { 1982, } 1992\end{array}$ \\
\hline 60 & Smith Tank Site & CC:5:6 & 675 & $\begin{array}{l}1275 \text { AD (Pueblo } \\
\text { III) }\end{array}$ & Ceramics & Woodson, 2007 \\
\hline 61 & Smoking Pipe & $\begin{array}{l}\text { NeotomaDB } \\
\quad 6363 ; 42 \mathrm{UT} 150\end{array}$ & 621 & $640 \pm 110$ yr BP & Bison bison bone & Billat, 1985 \\
\hline 62 & $\begin{array}{l}\text { Snake Rock } \\
\text { Village }\end{array}$ & $\begin{array}{l}\text { NeoMap 2851; } \\
\text { NeotomaDB } \\
6194\end{array}$ & 736 & $1,500 \pm 95$ yr BP & Wood & Aikens, 1967 \\
\hline 63 & Snaketown & - & 1,433 & $\begin{array}{r}835 \pm 109 ; 1,799 \\
\quad \pm 342 \text { cal yr BP }\end{array}$ & Ceramics & $\begin{array}{l}\text { Haury, 1965; } \\
\text { Agenbroad and } \\
\text { Haynes, } 1975\end{array}$ \\
\hline 64 & Spotten Cave & $\begin{array}{l}\text { 42UT104, } \\
\text { NeotomaDB } \\
6358\end{array}$ & 684 & $730 \pm 90$ yr BP & - & Cook, 1980 \\
\hline 65 & Stanton's Cave ${ }^{c}$ & $\begin{array}{l}\text { C:5:3; NAU QSP } \\
\quad \text { Site 9121; } \\
\text { GRCA } \\
\text { Accession 4597; } \\
\text { NeotomaDB } \\
\text { 5747; NeoMap } \\
\text { 2008 \& } 2004\end{array}$ & 14,191 & $\begin{array}{l}5,760 \pm 200 \\
\quad 17,300 \pm 800 \\
\text { yr BP }\end{array}$ & $\begin{array}{l}\text { Oreamnos } \\
\quad \text { harringtoni } \\
\text { dung }\end{array}$ & $\begin{array}{l}\text { Harington and } \\
\text { Euler, 1984; } \\
\text { Martin, 1984; } \\
\text { Mead and } \\
\text { Agenbroad, } \\
\text { 1989, } 1992\end{array}$ \\
\hline
\end{tabular}


TABLE 1- $\square$ Continued.

\begin{tabular}{|c|c|c|c|c|c|c|}
\hline Key & Locality name & $\begin{array}{l}\text { Database } \\
\text { reference }\end{array}$ & $\begin{array}{l}\text { Mean age } \\
(\text { cal yr BP) }\end{array}$ & Cited age & Dating method & References \\
\hline 66 & Sudden Shelter & $\begin{array}{r}\text { NeoMap 2225; } \\
\text { NeotomaDB } \\
5856 ; 45 \mathrm{SV} 6\end{array}$ & 7,458 & $\begin{array}{l}6,310 \pm 240 \\
\quad 7,090 \pm 85 \mathrm{yr} \\
\quad \mathrm{BP}\end{array}$ & Charcoal & $\begin{array}{l}\text { Lucias and } \\
\quad \text { Colville, } 1980\end{array}$ \\
\hline 67 & $\begin{array}{c}\text { Texas Creek } \\
\text { Overlook }\end{array}$ & $\begin{array}{l}\text { NeoMap 1148; } \\
\text { NeotomaDB } \\
\text { 5197; 5RB2435 }\end{array}$ & 458 & $430 \pm 50 \mathrm{yr} \mathrm{BP}$ & Charcoal & $\begin{array}{l}\text { Creasman and } \\
\text { Scott, } 1987\end{array}$ \\
\hline 68 & $\begin{array}{l}\text { Upper Sand } \\
\text { Island Site }\end{array}$ & - & 12,000 & $\begin{array}{l}13,000-11,000 \mathrm{cal} \\
\text { yr BP }\end{array}$ & Carving styles & $\begin{array}{l}\text { Malotki and } \\
\text { Wallace, } 2011\end{array}$ \\
\hline 69 & Walnut Canyon $^{\mathrm{a}}$ & - & 750 & $\begin{array}{r}1150-1220 \mathrm{AD} \\
\text { (Pueblo III) }\end{array}$ & Ceramics & Starkovich, 2011 \\
\hline 70 & Walton Creek ${ }^{\mathrm{a}}$ & $\begin{array}{l}\text { NeotomaDB } \\
\text { 5202; 5RT11 }\end{array}$ & 1,672 & $\begin{array}{l}1,730 \pm 225 \mathrm{yr} \\
\mathrm{BP}\end{array}$ & Charcoal ${ }^{14} \mathrm{C}$ & O’Neil, 1980 \\
\hline 71 & Wetherill Mesa & $\begin{array}{l}\text { NeoMap 1282; } \\
\text { NeotomaDB } \\
\text { 5316; Site } 1644\end{array}$ & 1,237 & $\begin{array}{l}1,237 \pm 34 \mathrm{cal} \mathrm{yr} \\
\mathrm{BP}\end{array}$ & $\begin{array}{l}\text { Building } \\
\text { foundation } \\
\text { style }\end{array}$ & $\begin{array}{l}\text { Hayes and } \\
\quad \text { Lancaster, } 1975\end{array}$ \\
\hline 72 & Whiskey Creek & $\begin{array}{l}\text { NeoMap 2438; } \\
\text { NeotomaDB } \\
\text { 5968; LA } 4986\end{array}$ & 1,540 & $\begin{array}{l}600 \pm 32 ; 2,310 \\
\pm 50 \text { cal yr BP } \\
\text { (Pueblo III - } \\
\text { Early } \\
\text { Basketmaker } \\
\text { II) }\end{array}$ & - & Heller, 1976 \\
\hline 73 & Wither's Wallow & $\begin{array}{l}\text { GLCA Accession } \\
\text { 82; NAU QSP } \\
\text { Site } 883\end{array}$ & 13,900 & $\begin{array}{l}12,010 \pm 160 \mathrm{yr} \\
\mathrm{BP}\end{array}$ & Mammuthus dung & $\begin{array}{l}\text { Mead and } \\
\text { Agenbroad, } \\
\text { 1989, } 1992\end{array}$ \\
\hline 74 & Zion & ZION 12396 & 14,000 & Rancholabrean & $\begin{array}{l}\text { Pleistocene } \\
\text { gravels }\end{array}$ & $\begin{array}{l}\text { Smith and } \\
\text { Santucci, 2001; } \\
\text { J. Hall, Zion } \\
\text { NPS, pers. } \\
\text { comm. } 2013 \text {. }\end{array}$ \\
\hline
\end{tabular}

\footnotetext{
a Taxonomic reclassification from Bos taurus to Bison bison.

b Taxonomic classification from "Unknown" to Bison sp.

c Taxonomic reclassification from Equus sp. to Bison sp.
}

Each Bison locality on the CP is listed in Table 1 and is numbered consecutively to correspond with the location shown in Figure 1. Undocumented and misidentified specimens from archaeological and paleontological localities are critical to our assessment. These specimens are archived in collections at the Museum of Northern Arizona (MNA), at the Grand Canyon NP, and at the East Tennessee State University Vertebrate Paleontology lab. The previously undescribed specimens consist predominately of dung and skeletal remains from cave, rock shelter, and packrat midden localities. We verified theidentification of each specimen and, if necessary, corrected it using Balkwill and Cumbaa (1992), who improved upon identification guides by Lawrence (1951) and Olsen (1960). In an attempt to capture the full extent of variation, Balkwill and Cumbaa (1992) included every postcranial element and provide 192 quantifiable, osteological characters for comparing Bison, represented by 27 individuals of B. bison bison and B. bison athabascae, and Bos taurus, represented by 16 individuals of several breeds including Holstein, Ayrshire, Shorthorn, Longhorn, and Africander. Balkwill and Cumbaa (1992) described specimens of both sexes and of various ages to account for natural variation within Bison and Bos.
Occurrences of Bison were also recovered from the following online data sets: Neotoma Paleoecology Database (NeotomaDB, www.neotomadb.org); Arizona's Cultural Resource Inventory (AZSite, www.azsite.asurite.ad.asu.edu/azsite/); and Neogene Mammal Mapping Portal (NeoMap, www.ucmp. berkely.edu/neomap/). Several localities were in the legal format of Township and Range, which creates a large polygon instead of a point and is imprecise when using point data for other site localities. For each site that was in the legal format, we converted the data into the coordinate system by using a centralized datum in the Township and Range overlay (B. Clark, Earth Point: Township and Range-Public Land Survey System on Google Earth, www.earthpoint.us.; Earth Point Corporation, Kuna, Idaho) in Google Earth Pro (Google Earth Pro, version 7.1.5.1557; Google Inc., Googleplex, Mountain View, California) using decimal degrees. The location is less accurate but more precise for geospatial assessment and analysis.

Each locality has a name and numbering system used by the archiving institution and maintained here. As part of this research, we directly radiocarbon dated Bison specimens from two sites, B:16:0461 and Grand Falls. For other sites, the age is 


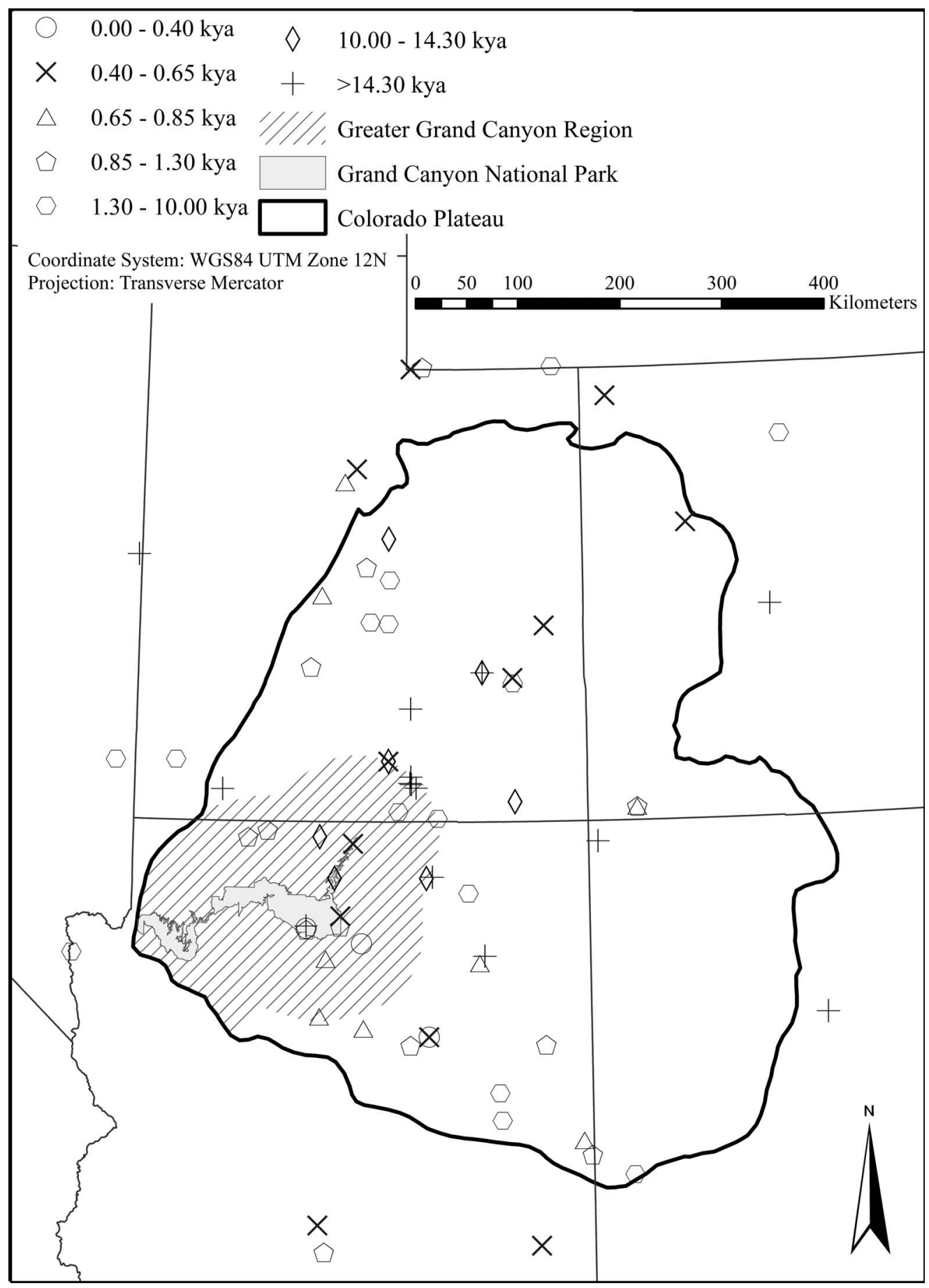

FIG. 2-Map of Bison localities placed in chronological context on the Colorado Plateau (CP, heavy black line) and surrounding region. Hashed line represents our definition of the Greater Grand Canyon Region (GGC; see text). Time unit is in thousands of calendar years ago BP (kya).

first given as reported in the literature (if provided) or archival database. We converted the given age(s) to calendar years BP as a single mean geologic age for geospatial representation (Fig. 2). The calibration of the radiocarbon years was completed using OxCal Online Tool (https://c14.arch.ox.ac.uk/) by employing the IntCal13 curve (Reimer et al., 2013). We assumed the average date of the calibrated age to be accurate, but it should be understood to be imprecise. We provide details including pertinent published references, archival databases, and dating sources for each site when available.

North American Quaternary Chronologies-There are a number of chronologies that are defined by specific metrics and cannot be easily integrated because of the inherent differences of paleontological and archaeological definitions; we define these below (Fig. 3). Each chronology used here is necessary for relating our various paleozoological sites that 


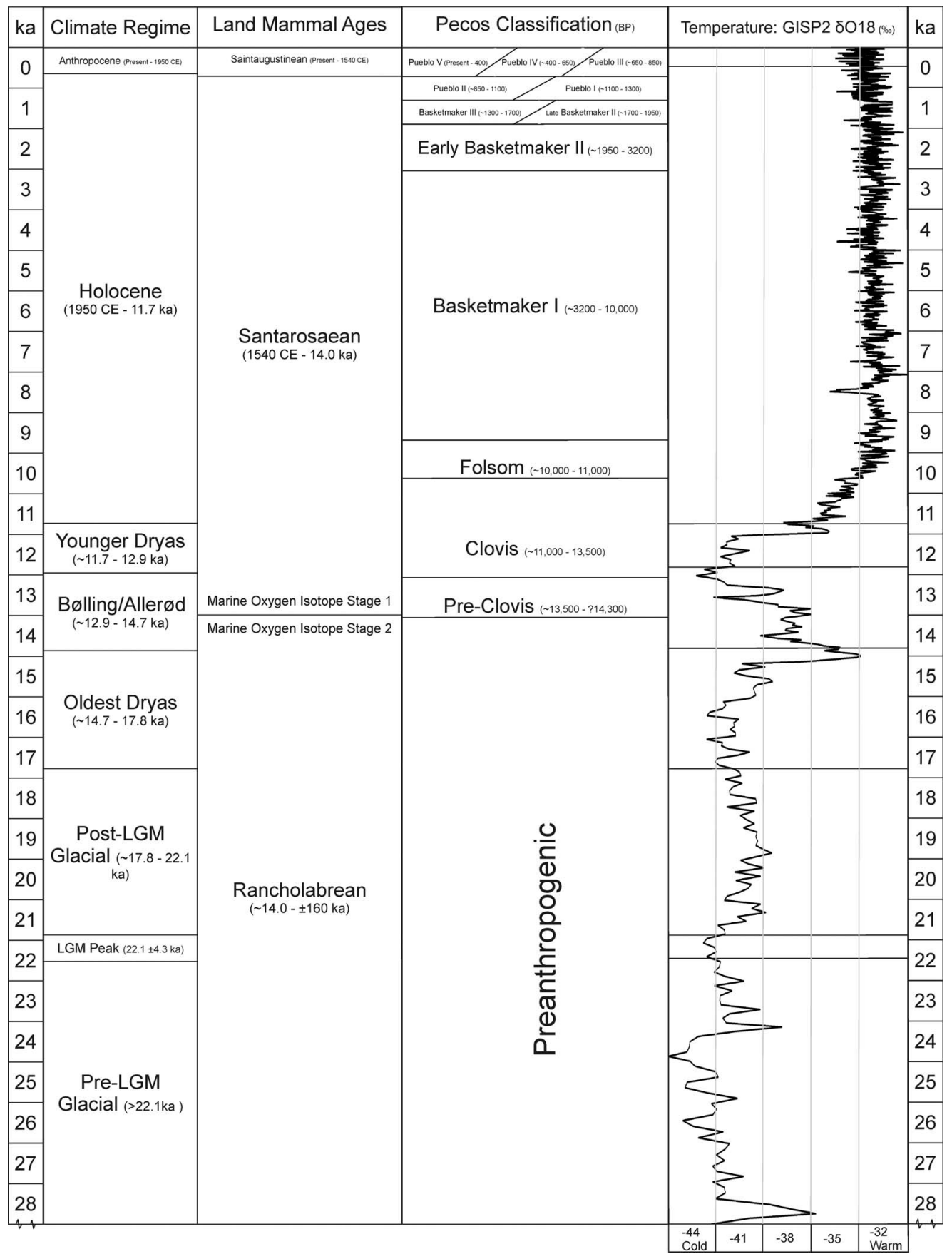

FIG. 3-Integrated North American Quaternary chronology. This time scale displays geological, paleontological (North American Land Mammal Ages: [Bell et al., 2004; Barnosky et al., 2014]), climatic (Climate Regime: Zhao et al., [2005]; Cohen et al., [2013]), and cultural (Pecos Classification: Polyak and Asmerom [2001]) units that are all important for relating Bison localities. Not all units are displayed to scale; some cultural units are enlarged for legibility. The relative temperature (solid line) is represented as a proxy derived from 818 -Oxygen isotopes from the Greenland Ice Sheet Project (GISP2). Data from Marine Oxygen Isotope Stages (Anderson et al., 2000); and temperature from GISP2 (Grootes et al., 1993). Abbreviations: ka = kilo annum (thousand years ago); BP, Before Present; CE, Common Era; \%o, part per thousand.

contain Bison remains. Furthermore, we implemented a climatic representation for understanding the environmental context of each period.

The Pleistocene is divided into biochronological units referred to as North American Land Mammal Ages based on faunal assemblage components. The Rancholabrean Land
Mammal Age is defined by the first appearance of Bison south of $55^{\circ} \mathrm{N}$ latitude and begins approximately 160,000 years BP (Bell et al., 2004). Furthermore, the Rancholabrean Land Mammal Age terminates 14,000 years BP when the Santarosaean Land Mammal Age begins (see discussion in Barnosky et al., 2014, for Land Mammal Age divisions within the Holocene). 
TABLE 2-Temporal summary of Bison localities on the Colorado Plateau. "BP" is calendar years Before Present.

\begin{tabular}{lllr}
\hline \hline \multicolumn{1}{c}{ Years BP } & Pecos classification & Geologic time & Bison localities $(n)$ \\
\hline $160,000-14,300$ & Preanthropogenic & Late Pleistocene & 14 \\
$14,300-10,000$ & PaleoIndian & Latest Pleistocene & 8 \\
$10,000-1,300$ & Archaic & Early to late Holocene & 15 \\
$1,300-850$ & Pueblo I-II & Latest Holocene & 14 \\
$850-650$ & Pueblo II & Latest Holocene & 8 \\
$650-400$ & Pueblo IV & Latest Holocene & 12 \\
$400-$ Present & Pueblo V & Latest Holocene & 3 \\
\hline
\end{tabular}

In addition to the mammal ages, archaeological stages were implemented based on North American human cultures in the Southwest (Polyak and Asmerom, 2001). The Pecos Classification was used in this study because it is culturally specific to the $\mathrm{CP}$ region and to the Southwest, and it often relates to the specimens we observed in museum collections. The later subdivisions of the Pecos Classification are within modern times. For this study, 850 years BP to today, which includes the formal historic, prehistoric, and protohistoric periods, is considered modern times to illustrate Bison nativity both precontact and postcontact with Europeans in the Southwest.

RESUlts-Herein, we report 74 sites located on or near the CP (with a few on the periphery) that contain Bison. We add 26 localities to the 48 reported in a recent study by Huffer (2013). Moreover, the previous study found that only 16 localities were described from the Holocene, of which 10 were on the CP (Huffer, 2013). In contrast to the Huffer (2013) study, we found 52 sites from the Holocene, of which 40 were on the CP.

Our assessment resulted in the summary data presented in Table 2. We present two sites of interest in and near Grand Canyon NP-B:16:0461 (specimen GRCA 69396) and Grand Falls (specimen MNA V8301; Table 1). We selected these specimens because they were previously insufficiently identified and only relatively dated.

Site B:16:0461, in the Mather Campground area of Grand Canyon NP, was a surface collection that had not been previously reported or identified. It is a lateral half of a proximal phalanx that is identified as Bison sp. because the lateral margin is curved (see Balkwill and Cumbaa (1992) and Martin (2014) for discussion). This sample returned a radiocarbon date (Beta 374436) of 50 \pm 30 years $\mathrm{BP}$ (measured radiocarbon age of $100.5 \pm 0.4$ $\mathrm{pMC})$.

The Grand Falls specimens represent two nearly complete postcranial individuals deposited $15 \mathrm{~m}$ apart in the same arroyo. In collections, these specimens' elements are mostly joined together with consolidant and adhesive. Here, we only describe the right astragalus because it was not treated with consolidant or adhesive. The medial tubercle of the astragalus is on a level with or above the line drawn across the proximal margin of the distal trochlea, as elaborated in Balkwill and Cumbaa (1992). The posterior surface of the astragalus is excavated and extends as far as the lateral margin. Grand
Falls was described in the MNA computer database as an arroyo site near the Little Colorado River, which suggests that it is possibly of Pleistocene age. However, radiocarbon dating (Beta 374435) returned an age of $50 \pm 30$ years BP (measured radiocarbon age of 102.4 \pm 0.4 pMC). Subsequently, we placed both B:16:0461 and Grand Falls at the beginning of the 20th century (Pueblo V).

DisCUSSION-Much of what we know about bison on the $\mathrm{CP}$ is based on historical and modern studies. Although these are valuable resources, they are incomplete and do not explore the prehistoric record. We found that 13 of our newly identified 26 Bison localities (50\%) were either previously not identified as Bison or were incorrectly identified as "unknown," "large mammal," or "Bos." Previous studies rarely identified Bison remains from in situ, pre-European contact because it was traditionally thought that Bison did not inhabit the CP. We are particularly intrigued that some researchers identified an in situ, pre-European contact faunal remain as "Bos taurus," knowing that this taxon arrived with the exploration of Spanish conquistadors, approximately 1540 Common Era. We hope to dispel this persistent line of circular reasoning by providing a thorough assessment of Bison on the CP since the latest Pleistocene.

Some hypothesize that the bison found in the Greater Grand Canyon Region were carrion from scavengers, bloat-and-float from upstream Colorado River, or goods traded by peoples. However, it would seem that the preColombian cultural trading of Bison elements might not have been traded long distances but more locally (Cannon, 2001). Reed (1952, 1955) points out that the bison skeletal and artifactual remains found in a preSpanish context across the southern CP (Arizona) are likely due to trade connections; albeit, Reed does state that the record of bison ("cibola") provided by the Havasupai Indians in the Grand Canyon probably represents a local procurement and not a trade item (independently described in Garcés and Coues, 1900:403, 406).

In addition, Bison are not known to frequent caves and are categorized as low frequenters of such shelters, with no more than $16.9 \%$ of all Bison remains found in caves across the contiguous United States (Jass and George, 
2010). Thus, finding Bison remains in caves or rock shelters at 45 of $74(60.8 \%)$ sites on the CP appears to be significant because either they were inhabiting the area or they were scavenged from a nearby location. For comparison, approximately $85.7 \%$ of bighorn sheep (Ovis canadensis) remains are found in caves (Jass and George, 2010).

ConCLUSION-The direct impact of this study is the production of the first comprehensive review of late Pleistocene and Holocene Bison on the Colorado Plateau. The results indicate that the geographic range of fossil and modern Bison can be extended to include the CP. Our data imply that Bison have been part of the CP from at least approximately 44,000 radiocarbon years ago though the latest Pleistocene (Rancholabrean) to the onset of the Holocene $(\sim 11,000$ years ago). The past approximately 11,700 years is a critical time because it marks the beginning of the modern climate based on paleobotanical records (McClaran and Van Devender, 1995; Coats et al., 2008). Major ecological and faunal turnover occurred at or by approximately 11,700 years ago, yet the early Holocene climate was still colder than today. The fossil Bison record for the early Holocene is not well-reported on the CP, but there appears to be good evidence of Bison presence during the late Holocene, through the various cultural phases, and up to the time of European contact. The southern CP (south of the Grand Canyon) in Arizona appears to have been fairly devoid of bison; but, within the Grand Canyon (likely just north of the Colorado River) and north, Bison were likely present, albeit never likely abundant based on its record. Though Bison were not overly common, such as observed on the Plains, the fossil record clearly demonstrates that this iconic bovid played a role in the biotic communities over much, if not all, of the Colorado Plateau up to the time of European contact.

We thank S. L. Swift and M. Carpenter for the countless hours contributed to data collection and improvement of this project. We thank the National Park Service (ZION 12396, Zion National Park Museum Collection) for the courtesy of abundant information and a specimen approved for destructive radiocarbon analysis (GRCA 69396) from the Grand Canyon National Park (research permit \# GRCA-2013-SCI-0052), and C. Hyde and B. Holton for informative discussions about the House Rock Valley bison herd. We thank the Navajo Nation, who in conjunction with the Museum of Northern Arizona provided their specimen for destructive radiocarbon analysis MNA V8301, Loc. 1104 (Navajo Nation Minerals Department research permit date: 30 August 2013). We thank many staff members of the Museum of Northern Arizona for their assistance. S. Emslie and M. Stiger provided important information for bison presence in the Gunnison Basin. We thank the National Buffalo Foundation, the Dr. Ken Throlson American Buffalo Scholarship, Experiment.com (a crowd-sourcing platform), the Western Bison Association, the East Tennessee State University (ETSU) Don Sundquist Center of Excellence in Paleontology, and the ETSU
Graduate School Graduate Student Research Grant for providing funds to JMM. We appreciate the continued support of The Mammoth Site and contribution of anonymous reviewers for much improvement of the manuscript.

\section{Literature Cited}

Agenbroad, L. D., and C. V. Haynes. 1975. Bison bison remains at Murray Springs, Arizona. Kiva 40:309-313.

Aikens, C. M. 1967. Excavations at Snake Rock Village and the Beaver River No. 2 site. University of Utah Anthropological Papers 87:1-78.

Anderson, E. 1966. Animal remains. Pages 113-117 in Contributions to Mesa Verde archaeology III: Site 866 and the cultural sequence at four villages in the Far View Group, Mesa Verde National Park, Colorado (R. H. Lister, editor). University of Colorado Studies Series in Anthropology, No. 12, Boulder.

Anderson, R. S., J. L. Betancourt, J. I. Mead, R. H. Hevly, and D. P. ADAм. 2000. Middle- and late-Wisconsin paleobotanic and paleoclimatic records from the southern Colorado Plateau, USA. Palaeogeography, Palaeoclimatology, Palaeoecology 155:31-57.

Balkwill, D. M., and S. L. Cumbaa. 1992. A guide to the identification of postcranial bones of Bos taurus and Bison bison. Canadian Museum of Nature, Ottawa.

Barnosky, A. D., M. Holmes, R. Kirchholtes, E. Lindsey, K. C. Maguire, A. W. Poust, M. A. Stegner, J. Sunseri, B. Swartz, J. Swift, N. A. Villavicencio, and G. O. Wogan. 2014. Prelude to the Anthropocene: two new North American Land Mammal Ages (NALMAs). The Anthropocene Review 1:225-242.

Bell, C. J., E. Lundelius, JR., A. D. Barnosky, R. W. Graham, E. H. Lindsay, D. R. Ruez, JR., H. Semken, JR., S. D. Webb, and R. J. ZAKRSEWSKI. 2004. The Blancan, Irvingtonian, and Rancholabrean Mammal Ages. Pages 232-314 in Late Cretaceous and Cenozoic mammals of North America: biostratigraphy and geochronology (M. O. Woodburne, editor). Columbia University Press, New York.

BiLlat, S. E. 1985. A study of Fremont subsistence at the Smoking Pipe site. M.S. thesis, Brigham Young University, Provo, Utah.

Blakey, R. C., And W. Ranney. 2008. Ancient landscapes of the Colorado Plateau: Grand Canyon. Grand Canyon Association, Grand Canyon, Arizona.

Broughton, J. M., D. A. Byers, R. A. Bryson, W. Eckerle, and D. B. Madsen. 2008. Did climatic seasonality control late Quaternary artiodactyl densities in western North America? Quaternary Science Reviews 27:1916-1937.

Bryan, A. L. 1979. Smith Creek Cave. Pages 164-251 in The archaeology of Smith Creek Canyon, eastern Nevada (D. R. Tuohy and D. L. Rendall, editors). Nevada State Museum Anthropological Papers, No. 17, Carson City.

Cannon, K. 2001. What the past can provide: contribution of prehistoric bison studies to modern bison management. Great Plains Research: A Journal of Natural and Social Sciences 11:145-174.

Coats, L. L., K. L. Cole, and J. I. MEad. 2008. 50,000 years of vegetation and climate history on the Colorado Plateau, Utah and Arizona, USA. Quaternary Research 70:322-338.

Cohen, K., S. Finney, P. Gibbard, and J. Fan. 2013. The ICS international chronostratigraphic chart. Episodes 36:199204.

Cоок, C. W. 1980. Faunal analysis from five Utah Valley sites: a 
test of a subsistence model from the Sevier Fremont Area. M.S. thesis, Brigham Young University, Provo, Utah.

Craine, J. M. 2013. Long-term climate sensitivity of grazer performance: a cross-site study. PLoS ONE 8:e67065.

Craine, J. M., E. Towne, D. Tolleson, and J. Nippert. 2013. Precipitation timing and grazer performance in a tallgrass prairie. Oikos 122:191-198.

Creasman, S., AND L. J. Scott. 1987. Texas Creek overlook: evidence for late Fremont (post A.D.1200) occupation in northwest Colorado. Southwestern Lore 53:1-16.

Cronin, M. A., M. D. MacNeil, N. Vu, V. Leesburg, H. D. Blackburn, and J. N. Derr. 2013. Genetic variation and differentiation of bison (Bison bison) subspecies and cattle (Bos taurus) breeds and subspecies. Journal of Heredity 104:500-509.

Downum, C. E., L. S. Cummings, K. Puseman, D. H. Sorrell, and M. M. VANCE. 2011. Archaeological excavations at Site B:16:105, Grand Canyon National Park. Archaeological Report 1247, Northern Arizona University, Flagstaff.

Emslie, S. D. 1987. Age and diet of fossil California condors in Grand Canyon, Arizona. Science 237:768-770.

EmsLie, S. D. 1988. Vertebrate paleontology and taphonomy of caves in Grand Canyon, Arizona. National Geographic Research 4:128-142.

Emslie, S. D. 2002. Fossil shrews (Insectivora: Soricidae) from the late Pleistocene of Colorado. Southwestern Naturalist 47:6269.

Flores, D. 1991. Bison ecology and bison diplomacy: the southern plains from 1800 to 1850. Journal of American History 78:465-485.

Fowler, D. D., D. B. Madsen, and E. M. Hattori. 1973. Prehistory of southeastern Nevada. University of Nevada Desert Research Institute Publications in the Social Sciences, No. 6, Reno.

FrIson, G. C. 1978. Prehistoric hunters of the high plains. Academic Press, New York.

Garcés, F. T. H., AND E. Coues. 1900. On the trail of a Spanish pioneer: the diary and itinerary of Francisco Garcés (missionary Priest) in his travels through Sonora, Arizona, and California, 1775-1776. F.P. Harper, New York.

Gillette, D. D., AND D. B. Madsen. 1992. The short-faced bear Arctodus simus from the late Quaternary in the Wasatch Mountains of central Utah. Journal of Vertebrate Paleontology 12:107-112.

Gillin, J., AND G. M. Allen. 1941. Archaeological investigations in central Utah. Papers of the Peabody Museum of Archaeology and Ethnology, Harvard Museum 17:1-46.

Grootes, P., M. Stuiver, J. White, S. Johnsen, and J. Jouzel. 1993. Comparison of oxygen isotope records from the GISP2 and GRIP Greenland ice cores. Nature 366:552-554.

Hansen, R. M. 1980. Late Pleistocene plant fragments in the dungs of herbivores at Cowboy Cave in Cowboy Cave. Pages 179-189 in Cowboy Cave (J. D. Jennings, editor). University of Utah Anthropological Papers, No. 104, Salt Lake City.

Harington, C. R., AND R. Euler. 1984. Ungulate remains from Stanton's Cave: an identification list. Pages 69-75 in The archaeology, geology, and paleobiology of Stanton's Cave: Grand Canyon National Park, Arizona (R. C. Euler, editor). Grand Canyon Natural History Association, Grand Canyon, Arizona.

HARRIS, A. H. 2014. Pleistocene vertebrates of southwestern USA and northwestern Mexico. University of Texas at El Paso, El Paso.

HaurY, E. W. 1940. Excavations in the Forestdale Valley, eastcentral Arizona. University of Arizona Social Science Bulletin, No. 12, Tucson.

Haury, E. W. 1965. Food: animal. Pages 156-158 in Excavations at Snaketown: material culture (H. S. Gladwin, E. W. Haury, E. B. Sayles, and N. Gladwin, editors). University of Arizona Press, Tucson.

HAY, O. P. 1927. On Pleistocene geology of western region of North America and remains of vertebrates occurring in it: Arizona. Pages 129-137 in The Pleistocene of the western region of North America and its vertebrated animals (O. P. Hay, editor). Publication No. 322B, Carnegie Institution, Washington, D.C.

Hayes, A. C., and J. A. Lancaster. 1975. Badger House community, Mesa Verde National Park. U.S. Department of the Interior, National Park Service, Wetherill Mesa Studies, Publications in Archeology 7E.

Heaton, T. H. 1988. Bears and man at Porcupine Cave, Western Uinta Mountains, Utah. Current Research in the Pleistocene 5:71-73.

Heller, M. M. 1976. Zooarchaeology of Tularosa Cave, Catron County, New Mexico. M.S. thesis, University of Texas at El Paso, El Paso.

Hesse, I., W. Parry, and F. Smiley. 1999. Badger Springs: a LatePaleoindian site in northeastern Arizona. Current Research in the Pleistocene 16:27-30.

Hoffmeister, D. 1986. Mammals of Arizona. University of Arizona Press, Tucson.

Hornaday, W. T. 1889. The extermination of the American bison. Smithsonian Institution, Government Printing Office, Washington, D.C.

HufFer, D. J. 2013. A spatial analysis and zooarchaeological interpretation of archaeological bison remains in the Southwest and the wildlife management implications for the House Rock Valley bison herd in Grand Canyon National Park, Arizona. M.A. thesis, The University of Arizona, Tucson.

IsENBERG, A. C. 1997. The returns of the Bison: nostalgia, profit, and preservation. Environmental History 2:179-196.

Jass, C. N., AND C. O. George. 2010. An assessment of the contribution of fossil cave deposits to the Quaternary paleontological record. Quaternary International 217:105116.

Jennings, J. D. 1978. Prehistory of Utah and the eastern Great Basin. University of Utah Anthropological Papers 98:1-279.

Johnson, P. C. 1974. Mammalian remains from Los Colinas, a classic Hohokam site in Arizona. M.S. thesis, University of Arizona, Tucson.

Jones, A. T. 1986. A cross section of Grand Canyon archaeology: excavations at five sites along the Colorado River. Publications in Anthropology No. 28. Western Archaeological and Conservation Center, National Park Service, Tucson, Arizona.

Koch, P. L., AND A. D. Barnosky. 2006. Late Quaternary extinctions: state of the debate. Annual Review of Ecology, Evolution, and Systematics 37:215-250.

Kropf, M., J. I. Mead, and R. S. Anderson. 2007. Dung, diet, and the paleoenvironment of the extinct shrub-ox (Euceratherium collinum) on the Colorado Plateau, USA. Quaternary Research 67:143-151.

LAWRENCE, B. 1951. Mammals found at the Awatovi Site: postcranial skeletal characters of deer, pronghorn, and sheep- 
goat with notes on Bos and Bison. Papers of the Peabody Museum of Archaeology and Ethnology, Harvard University 35:1-43.

LEONARD, R. D. W. 1989. Anasazi faunal exploitation: prehistoric subsistence on northern Black Mesa, Arizona. Southern Illinois University Center for Archaeological Investigations Occasional Paper, No. 13, Carbondale.

Lightfoot, K. G., and G. M. Feinman. 1982. Social differentiation and leadership development in early pithouse villages in the Mogollon region of the American Southwest. American Antiquity 47:64-86.

Lindsay, E. E. H., AND N. T. N. Tessman. 1974. Cenozoic vertebrate localities and faunas in Arizona. Journal of the Arizona Academy of Science 9:3-24.

Lucas, S. G., T. Williamson, and J. Sobus. 1988. Late Pleistocene (Rancholabrean) mammals from the Edith Formation, Albuquerque, New Mexico. New Mexico Journal of Science 28:51-58.

Lucias, W. A. 1980. Bone and shell material. Pages 97-107 in Cowboy Cave (J. D. Jennings, editor). University of Utah Anthropological Paper, No. 104, Salt Lake City.

LuCias, W. A., AND J. K. Colville. 1980. Osteological analysis of faunal remains. Pages $157-170$ in Sudden shelter (J. D. Jennings, A. R. Schroedl, and R. N. Holmer, editors). University of Utah Anthropological Papers, No. 103, Salt Lake City.

Madsen, D. B., D. Rhode, D. K. Grayson, J. M. Broughton, S. D. Livingston, J. Hunt, J. Quade, D. N. Schmitt, and M. W. Shaver III. 2001. Late Quaternary environmental change in the Bonneville Basin, western USA. Palaeogeography, Palaeoclimatology, Palaeoecology 167:243-271.

Malotki, E., and H. D. Wallace. 2011. Columbian mammoth petroglyphs from the San Juan River near Bluff, Utah, United States. Rock Art Research 28:143-152.

Martin, J. M. 2014. Late Pleistocene and Holocene Bison of Grand Canyon and Colorado Plateau: implications from the use of paleobiology for natural resource management policy. M.S. thesis, East Tennessee State University, Johnson City.

Martin, P. 1984. Stanton's Cave during and after the last ice age. Pages 133-137 in The archaeology, geology, and paleobiology of Stanton's Cave: Grand Canyon National Park, Arizona (R. C. Euler, editor). Grand Canyon Natural History Association, Grand Canyon, Arizona.

MarwitT, J. P. 1968. Pharo Village. University of Utah Anthropological Papers 91:1-99.

McClaran, M. P., and T. R. VAn Devender. 1995. The desert grassland. University of Arizona Press, Tucson.

McDonald, J. N. 1981. North American bison: their classification and evolution. University of California Press, Los Angeles.

McKern, S. 1988. Archaeological investigation at the Bessie Bottom site (48UT1186): late prehistoric animal procurement in the upper Bear River drainage. Cultural Resource Management Report, No. 45, Western Wyoming College, Rock Springs.

Mead, J. I., And L. D. Agenbroad. 1989. Pleistocene dung and the extinct herbivores of the Colorado Plateau, southwestern USA. Cranium 6:29-44.

Mead, J. I., and L. D. Agenbroad. 1992. Isotope dating of Pleistocene dung deposits from the Colorado Plateau, Arizona and Utah. Radiocarbon 34:1-19.

Mead, J. I., C. J. Bell, and L. K. Murray. 1992. Mictomys borealis (northern bog lemming) and the Wisconsin paleoecology of the east-central Great Basin. Quaternary Research 37:229238.

Mead, J. I., S. E. Sharpe, and L. D. Agenbroad. 1991. Holocene Bison from Arches National Park, southeastern Utah. Great Basin Naturalist 51:336-342.

Mead, J. I., R. S. Thompson, and T. R. Van Devender. 1982. Late Wisconsinan and Holocene fauna from Smith Creek Canyon, Snake River Range, Nevada. Transactions of the San Diego Society of Natural History 20:1-26.

Miller, W. E. 1987. Mammut americanum, Utah's first record of the American mastodon. Journal of Paleontology 61:168-183.

Montgomery, R. G., W. Smith, ANd J. O. Brew. 1949. Franciscan Awatovi: the excavation and conjectural reconstruction of a 17th-century Spanish mission establishment at a Hopi Indian town in northeastern Arizona. Papers of the Peabody Museum of American Archaeology and Ethnology, Harvard University $36: 1-361$.

O'NeIL, B. 1980. Highway salvage excavations at Walton Creek (5RT11), Routt County, Colorado. Southwestern Lore 46:3.

Olsen, S. J. 1960. Post-cranial skeletal characters of Bison and Bos. Papers of the Peabody Museum of Archaeology and Ethnology, Harvard University 35:1-15.

Osborn, A. J., J. Brown, G. Burgett, L. S. Cummings, R. J. Hartley, S. Vetter, J. Waters, and T. Zalucha. 1995. Aboriginal adaptations on the Colorado Plateau: a view from the Island-in-the-Sky, Canyonlands National Park, Utah. Midwest Archeological Center Occasional Studies in Anthropology 33:245-309.

Polyak, V. J., And Y. Asmerom. 2001. Late Holocene climate and cultural changes in the southwestern United States. Science 294:148-151.

Ranney, W. (editor). 2012. Carving Grand Canyon. Second edition. Grand Canyon Association, Grand Canyon, Arizona.

REED, E. K. 1952. The myth of Montezuma's bison and the type locality of the species. Journal of Mammalogy 33:390-392.

Reed, E. K. 1955. Bison beyond the Pecos. Texas Journal of Science 7:130-135.

ReImer, P., E. BARD, AND A. Bayliss. 2013. IntCal13 and Marine13 radiocarbon age calibration curves $0-50,000$ years cal $\mathrm{BP}$. Radiocarbon 55:1869-1887.

ReImONDO, E. L. 2012. Ecological impacts and management implications of introduced bison in the Grand Canyon. M.S. thesis, Northern Arizona University, Flagstaff.

Sanderson, E. W., K. H. Redford, B. Weber, K. E. Aune, D. Baldes, J. Berger, D. Carter, C. Curtin, J. N. Derr, S. Dobrott, E. Fearn, C. Fleener, S. Forrest, C. Gerlach, C. C. Gates, J. E. Gross, P. Gogan, S. Grassel, J. A. Hilty, M. Jensen, K. Kunkel, D. Lammers, R. List, K. Minkowski, T. Olson, C. Pague, P. B. Robertson, and B. Stephenson. 2008. The ecological future of the North American bison: conceiving long-term, large-scale conservation of wildlife. Conservation Biology 22:252-266.

ScotT, E., AND S. M. Cox. 2008. Late Pleistocene distribution of Bison (Mammalia; Artiodactyla) in the Mojave Desert of southern California and Nevada. Science Series 41:359-382.

Seager, R., N. Graham, C. Herweijer, A. L. Gordon, Y. Kushnir, AND E. CoOK. 2007. Blueprints for medieval hydroclimate. Quaternary Science Reviews 26:2322-2336.

Sharrock, F. W. 1966. Prehistoric occupation patterns in southwestern Wyoming and cultural relationships with the Great Basin and Plains cultural areas. University of Utah Anthropological Papers 77:1-234.

Smartt, R. A., S. G. LuCAS, AND D. J. Hafner. 1991. The giant bison 
(Bison latifrons) from the middle Rio Grande Valley of New Mexico. Southwestern Naturalist 36:136-137.

Smith, J., And V. SANtucci. 2001. Vertebrate ichnostratigraphy of the Glen Canyon Group (Jurassic) in Zion National Park, Utah. Pages 15-19 in The changing face of the east Mojave Desert and abstracts from the 2001 Desert Symposium (R. E. Reynolds, editor). California State University Desert Studies Consortium, Fullerton.

Starkovich, B. 2011. Faunal analysis of Walnut Canyon, Arizona. M.S. thesis, University of Arizona, Tucson.

Stein, W. 1963. Mammal remains from archaeological sites in the Point of Pines region, Arizona. American Antiquity 29:213220.

Teague, L. S., and W. L. Deaver. 1989. The 1982-1984 excavations at Las Colinas. Cultural Resource Management Division, Arizona State Museum, University of Arizona, Tucson

WAKELING, B. F. 2006. Arizona bison genetics: verifying origins. Pages 25-30 in Managing wildlife in the Southwest: new challenges for the 21st century (J. W. Cain III and P. R. Krausman, editors). A Publication of the Southwest Section of The Wildlife Society, Tucson, Arizona.
Walker, D. N. 1983. Faunal remains and subsistence practices at $5 \mathrm{MF} 605$, a fur trading post in northwest Colorado. Southwestern Lore 49:6-29.

Wisely, S., M. Statham, and R. Fleischer. 2008. Pleistocene refugia and Holocene expansion of a grassland-dependent species, the black-footed ferret (Mustela nigripes). Journal of Mammalogy 89:87-96.

Withers, K., AND J. MEAD. 1993. Late Quaternary vegetation and climate in the Escalante River basin on the central Colorado Plateau. Great Basin Naturalist 53:145-161.

WolfF, S. E. 2013. Home on the range: biogeographic distribution of bison in Arizona. PalArch's Journal of Vertebrate Palaeontology 10:1-11.

Woodson, K. 2007. The Goat Hill Site and ancestral Pueblo migrations into the Safford Basin. The Petroglyph, Newsletter of the Arizona Archaeological Society 43:7-11.

Zhao, M., C.-Y. Huang, and K.-Y. Wei. 2005. A 28,000 year U K37 sea-surface temperature record of ODP Site 1202B, the Southern Okinawa Trough. TAO 16:45-56.

Submitted 7 July 2016. Accepted 19 December 2016.

Associate Editor was Troy Ladine. 
Queries for swna-62-01-03

This manuscript/text has been typeset from the submitted material. Please check this proof carefully to make sure there have been no font conversion errors or inadvertent formatting errors. Allen Press. 